\title{
Molecular characteristics of Polish field strains of Marek's disease herpesvirus isolated from vaccinated chickens
}

\author{
Grzegorz Woźniakowski*, Elżbieta Samorek-Salamonowicz, Wojciech Kozdruń
}

\begin{abstract}
Background: Twenty-nine Marek's disease virus (MDV) strains were isolated during a 3 year period (2007-2010) from vaccinated and infected chicken flocks in Poland. These strains had caused severe clinical symptoms and lesions. In spite of proper vaccination with mono- or bivalent vaccines against Marek's disease (MD), the chickens developed symptoms of MD with paralysis.

Because of this we decided to investigate possible changes and mutations in the field strains that could potentially increase their virulence. We supposed that such mutations may have been caused by recombination with retroviruses of poultry - especially reticuloendotheliosis virus (REV).

Methods: In order to detect the possible reasons of recent changes in virulence of MDV strains, polymerase chain reaction (PCR) analyses for mea oncogene and for long-terminal repeat (LTR) region of REV were conducted. The obtained PCR products were sequenced and compared with other MDV and REV strains isolated worldwide and accessible in the GeneBank database.
\end{abstract}

Results: Sequencing of the meq oncogene showed a 68 basepair insertion and frame shift within 12 of 24 field strains. Interestingly, the analyses also showed $0.78,0.8,0.82,1.6 \mathrm{~kb}$ and other random LTR-REV insertions into the MDV genome in 28 of 29 of strains. These genetic inserts were present after passage in chicken embryo kidney cells suggesting LTR integration into a non-functional region of the MDV genome.

Conclusion: The results indicate the presence of a recombination between MDV and REV under field conditions in Polish chicken farms. The genetic changes within the MDV genome may influence the virus replication and its features in vivo. However, there is no evidence that meq alteration and REV insertions are related to the strains' virulence.

\section{Background}

Neoplastic diseases of poultry may be related to infection with Marek's disease virus (MDV) and with the group of avian retroviruses. MDV is a highly cellassociated herpesvirus with lymphotropic properties but its molecular and genomic structures are similar to Alphaherpesvirinae subfamily [1].

The MDV's genome consists of double stranded DNA about $180 \mathrm{~kb}$ and it is divided into unique long $\left(\mathrm{U}_{\mathrm{L}}\right)$ and unique short $\left(\mathrm{U}_{\mathrm{s}}\right)$ regions flanked by internal (ITR) and terminal (TR) repeats.

\footnotetext{
* Correspondence: grzegorz.wozniakowski@piwet.pulawy.pl National Veterinary Research Institute, Department of Poultry Viral Diseases, Partyzantow 57 Avenue, 24-100 Pulawy, Poland
}

The main oncoprotein of MDV is MEQ with leucine zipper characteristic for all transcriptional regulators and potential oncogens. MEQ is present in two copies located within the $\mathrm{TR}_{\mathrm{L}}$ region and between the junction of $I R_{L}$ and $I R_{s}$. Other MDV genes taking part in replication and oncogenesis are ICP4 (immediately-early promoter), pp38 (phosphoprotein from pp38-pp24 complex) and LAT (latency associated transcripts), which down regulate MDV replication during latency [2]. The MDV genome is known to be susceptible to recombination and insertion of parts of foreign viral genomes especially retroviruses like the avian leukosis virus (ALV) and reticuloendotheliosis virus (REV) [3-5].

Retroviruses are another group of oncogenic viruses. The Retroviridae family falls into a few genera. ALVs
C Biomed Central 
are classified into Alpharetrovirus [6] whereas the REV group is in the separate Gammaretrovirus genus. However the structure and organization of their genome are common. The genomes of retroviruses consist of singlestranded RNA with positive polarity and present in two copies.

REV is an immunosuppressive virus affecting both humoral and cellular immunity [7]. An additional problem with REV is their ability to integrate a part or entire genome into the DNA of MDV [3,8]. This phenomenon occurs under field conditions [9]. The influence of a LTR-REV insertion into the MDV genome may change viral oncogenity or attenuation.

The main objective of this study was to determine molecular characteristics of recently isolated Polish strains of MDV and to identify factors responsible for changes of MDV virulence; changes that are commonly associated with mutation of the nucleotide sequence of meq oncogene [10].

\section{Methods}

\section{Viruses}

A total of 29 MDV field strains isolated from spleens of spontaneously infected vaccinated chickens, the HPRS 16 reference strain (Houghton Poultry Research Station, Huntingdon. UK), the CVI988 Rispens strain from a commercial vaccine (Mérial, Lyon, France) and two BAC clones of Polish strains were propagated in chicken embryo kidney cells (CEKs) prepared from SPF chicken embryos (LTZ, Cuxhaven, Germany). A REV-T strain (National Veterinary Research Institute, Pulawy, Poland) was included in the study. The growth medium MEM (Gibco, Paisley, Scotland) supplemented with 10\% bovine foetal serum and 1\% antibiotics (Antibiotic-antimycotic, Gibco) was used, while a maintenance medium consisting of MEM with $1 \%$ mixture of antibiotics as above was used. MDV strains were incubated at $37.8^{\circ} \mathrm{C}$ in $5 \% \mathrm{CO}_{2}$ until a cytophatic effect (CPE) was observed. The REV-T strain was also cultivated in CEKs and harvested 5 days post inoculation Third passage of each strain in CEKs was suspended in preservative medium containing $10 \%$ DMSO, gradually chilled and finally frozen in liquid nitrogen. The strains are listed in Table 1. The purity of the MDV strains from REV contamination was tested by filtering supernatants from the $3^{\text {rd }}$ passage of each MDV strain through the $0.2 \mu \mathrm{M}$ syringe filters (Sartorious, Goettingen, Germany) and inoculating monolayers of CEKs. These cell cultures were incubated for 14 days in conditions as described above. Additionally DNA extracted from supernatants was tested with real-time polymerase chain reaction (real-time PCR) for the gag gene of REV according to the procedure developed by Hauck et al. [11] for excluding incidental contamination with REV.
Table 1 Marek's disease virus strains used in the study

\begin{tabular}{|c|c|c|}
\hline Number & Name of strain & Origin of strain \\
\hline 1 & 23_07_PL & 16-w layers, spleen \\
\hline 2 & 25_07_PL & 20-w breeders, spleen \\
\hline 3 & 29_07_PL & 16-w layers, spleen \\
\hline 4 & 30_07_PL & 17-w breeders, spleen \\
\hline 5 & 31_07_PL & 27-w layers, spleen \\
\hline 6 & 32_07_PL & 27-w layers, spleen \\
\hline 7 & 2_08_PL & 28-w layers, spleen \\
\hline 8 & 3_08_PL & 20-w layers, spleen \\
\hline 9 & 7_08_PL & 26-w layers, spleen \\
\hline 10 & 8_08_PL & 29-w layers, spleen \\
\hline 11 & 45_08_PL & 19-w breeders, spleen \\
\hline 12 & 48_08_PL & 15-w breeders, spleen \\
\hline 13 & 56_08_PL & 21-w layers, spleen \\
\hline 14 & 73_08_PL & 6-w broilers, spleen \\
\hline 15 & 8_09_PL & 5-w broilers, spleen \\
\hline 16 & 23_09_PL & 17-w layers, spleen \\
\hline 17 & 26_09_PL & 32-w layers, spleen \\
\hline 18 & 40_09_PL & 18-w breeders, spleen \\
\hline 19 & 42_09_PL & 7-w broilers, spleen \\
\hline 20 & 45_09_PL & 18-w layers, spleen \\
\hline 21 & 51_09_PL & 18-w layers, spleen \\
\hline 22 & 81_09_PL & 26-w layers, spleen \\
\hline 23 & 82_09_PL & 25-w layers, spleen \\
\hline 24 & 86_09_PL & 28-w layers, spleen \\
\hline 25 & 8_10_PL & 24-w layers, spleen \\
\hline 26 & 10_10_PL & 19-w layers, spleen \\
\hline 27 & 11_10_PL & 25-w layers, spleen \\
\hline 28 & 30_10_PL & 27-w layers, spleen \\
\hline 29 & 61_10_PL & 6-w broilers, spleen \\
\hline 30 & BAC_31_07 1 meq & $\begin{array}{l}\text { BAC-clone from Escherichia } \\
\text { coli GST1763 cells }\end{array}$ \\
\hline 31 & BAC_7_08 & $\begin{array}{l}\text { BAC clone from Escherichia } \\
\text { coli GST1763 cells }\end{array}$ \\
\hline 32 & $\mathrm{HPRS}_{16}$ & $\begin{array}{l}\text { Houghton Poultry Research Station, } \\
\text { Huntingdon UK }\end{array}$ \\
\hline 33 & CVl/988 Rispens & Commercial vaccine (Mérial) \\
\hline
\end{tabular}

All strains were passed 3 times in chicken embryo kidney cells.

W: week-old, CEK: chicken embryo kidney cells, BAC_31_07 $\Delta m e q$ : deletive strain of 31_07 strain in bacterial artificial chromosome.

\section{DNA extraction}

Total DNA was extracted from infected CEKs using QIAamp Mini Kit (Qiagen, Hilden, Germany) according to the manufacturer's procedure.

\section{Real-time PCR}

Primers complementary to ICP4, pp38 and meq oncogene were used [2]. For the normalisation of results as well as for calculation of viral copy number per $10^{4}$ infected cells, 
the chicken ovotransferrin gene was also amplified for each sample. Each sample was run in duplicate. Reaction mixture, standards and conditions of real-time PCR has been described elsewhere [2]. As positive controls, $\mathrm{HPRS}_{16}$ and Rispens strains as well as MDV BAC-clones were used.

\section{PCR}

PCR with primers complementary to meq was applied as previously described [10]. The PCR products were purified using Nucleospin Kit (Macherey-Nagel, Düren, Germany).

\section{PCR for LTR}

The sequences of primers specific for LTR-REV were: LTR U3 REV: 5'-CCG AGA AAT GAT ATC AGC-3' and U5 REV: 5-GGT GGG GTA GGG ATC CG-3' [9]. The reaction volume was $25 \mu \mathrm{L}$ and contained: $2.5 \mu \mathrm{L}$ of 10 -fold concentrated PCR buffer (1.5 $\mathrm{mM} \mathrm{MgCl} 2$ ), $1 \mu \mathrm{L}$ of dNTPs $(0.2 \mu \mathrm{M}$ of each dNTP), $0.5 \mu \mathrm{L}$ HotStar Taq Polymerase (2.5 U) (Qiagen), $5 \mu \mathrm{L}$ of $1 \mathrm{M}$ betaine solution $(0.2 \mu \mathrm{M})$ (Sigma-Aldrich, Munich, Germany), $1 \mu \mathrm{L}$ of each primer $(0.4 \mu \mathrm{M}), 1 \mu \mathrm{L}$ of total DNA and $13 \mu \mathrm{L}$ of deionised water. PCR conditions were as following: initial denaturation $-95^{\circ} \mathrm{C}$ for $5 \mathrm{~min}$., following 35 cycles of: exact denaturation $94^{\circ} \mathrm{C}$ for $1 \mathrm{~min}$., primers annealing $62^{\circ} \mathrm{C}$ for $1 \mathrm{~min}$., products extension $72^{\circ} \mathrm{C}$ $1 \mathrm{~min}$., final extension of the products $72^{\circ} \mathrm{C}$ for $10 \mathrm{~min}$. Positive control for PCR was extracted from CEKs inoculated with the REV-T strain while a negative control was extracted from non-inoculated cell cultures.

\section{DNA sequencing}

PCR products of meq from 24 MDV strains and 10 products for the LTR region were sequenced from forward and reversed primers on GS FLX/Titanium sequencer (Roche, Branford, Connecticut, USA) by GENOMED (Warsaw, Poland). Each amplicon was run 2 times to ensure the reliability of the results. The meq and LTR sequences were assembled into single contig in Bioedit ver. 7.0.9.0 then submitted to NCBI Genebank and aligned using Geneious ver. 5.1 [12]. PCR products were separated in $1.5 \%$ ethidium bromide stained agarose gels.

\section{Results}

As the first step of this study, the examined MDV field strains were propagated in CEKs. Three passages of these strains were conducted. CPE was observed in all infected cell cultures starting from the $96^{\text {th }} \mathrm{h}$ of $3^{\text {rd }}$ passage. An early CPE was seen as small round cells reflecting light waves. These cells formed foci and syncytias that detached from the wall of cell culture flask causing formation of plaques. From these passaged MDV strains, total DNA was extracted. Similarly, DNA extracted from
CEKs infected with REV-T was used as a positive control. There was no CPE observed in CEKs inoculated with filtered supernatants taken from cell cultures infected with MDV thus confirming the purity of the used strains. Similarly, the results of real-time PCR for the detection of gag gene of REV in supernatants were negative.

Next, real-time PCR was conducted on all DNA samples extracted from infected CEKs. The specific fluorescent signal observed as curves for ICP4, pp38 and meq genes were detected in all samples. On the basis of quantification of ICP4, pp38 and meq, the number of viral copies was determined as ranging from $63 \pm 2$ to $12123 \pm 587$ copies per $10^{4}$ infected cells.

\section{Meq analysis}

By standard PCR, the presence of an approximately $1062 \mathrm{bp}$ long product for the meq oncogene was found in samples except sample 73_08 (Figure 1, lane 14) and BAC_31_07 $\Delta$ meq (Figure 1, lane 30). In 5 samples (Figure 1, lanes: 1, 18, 19, 25 and 30) the PCR products were weak and for 7 samples, additional smears in the background were observed (Figure 1, lanes: 10, 11, 12, $16,25,26,30$ ) as fine bands ranging from 210 to 550 bp. PCR products for meq oncogene were sequenced and submitted to NCBI Genebank (Table 2).

Analysis of $m e q$ showed a 68 bp insertion in position 566 of $m e q$ in 12 of 24 strains (Figure 2). These insertions resulted in a frame shift in Polish field strains isolated in 2009 and 2010. Other minor single nucleotide

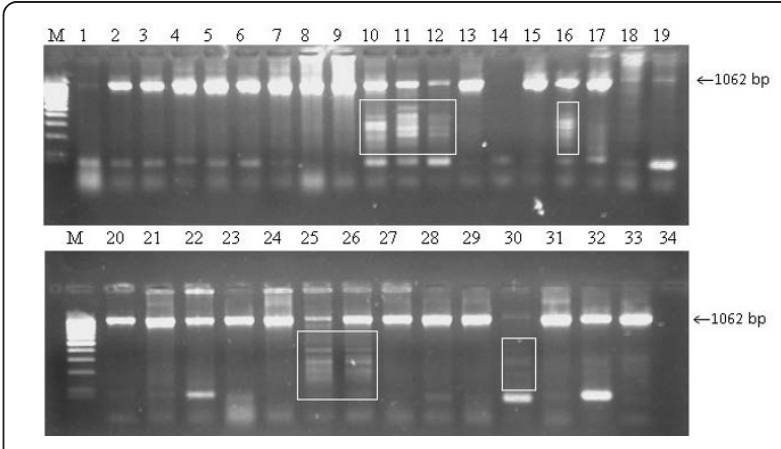

Figure $1 \mathrm{PCR}$ results for the detection of the meq oncogene in Marek's disease virus. M-Mass Ruller $100 \mathrm{~kb}$ Plus (80 bp-10 000 bp), Fermentas, 1 - 23_07_PL, 2 - 25_07_PL, 3 - 29_07_PL, 4 30_07_PL, 5 - 31_07_PL, 6 - 32_07_PL, 7 - 2_08_PL, 8 - 3_08_PL, 9 7_08_PL, 10 - 8_08_PL, 11 - 45_08_PL, 12 - 48_08_PL, 13 56_08_PL, 14 - 73_08_PL, 15 - 8_09_PL, 16 - 23_09_PL, 17 26_09_PL, 18 - 40_09_PL, 19 - 42_09_PL, 20 - 45_09_PL, 21 51_09_PL, 22 - 81_09_PL, 23 - 82_09_PL, 24 - 86_09_PL, 25 8_10_PL, 26 - 10_10_PL, 27 - 11_10_PL, 28 - 30_10_PL, 29 61_10_PL, 30 - BAC_31_07 1 meq, 31 - BAC_7_08, 32 - HPRS 16,33 Rispens, 34 - negative DNA control extracted from non-infected chicken embryo kidney cells. Smeared products are marked with a white box. 
Table 2 Sequences of meq oncogene and long terminal repeats sequence fragment of reticuloendotheliosis virus genome submitted to NCBI Genebank database

\begin{tabular}{llll}
\hline Strain & Accession number & Strain & Accession number \\
\hline 25_07_PL & Genebank:HQ204792 & 3_08_PL & Genebank:HQ204809 \\
\hline 8_10_PL & Genebank:HQ204793 & 7_08_PL & Genebank:HQ204810 \\
\hline 10_10_PL & Genebank:HQ204794 & 8_08_PL & Genebank:HQ204811 \\
\hline 11_10_PL & Genebank:HQ204795 & 12_08_PL & Genebank:HQ204812 \\
\hline 30_10_PL & Genebank:HQ204796 & 45_08_PL & Genebank:HQ204813 \\
\hline 8_09_PL & Genebank:HQ204797 & 56_08_PL & Genebank:HQ204814 \\
\hline 23_09_PL & Genebank:HQ204798 & 73_08_PL & Genebank:HQ204815 \\
\hline 40_09_PL & Genebank:HQ204799 & LTR 7_08 & Genebank:HQ204816 \\
\hline 42_09_PL & Genebank:HQ204800 & LTR 11_10 & Genebank:HQ204817 \\
\hline 45_09_PL & Genebank:HQ204801 & LTR 29_07 & Genebank:HQ204818 \\
\hline 51_09_PL & Genebank:HQ204802 & LTR 31_07 & Genebank:HQ204819 \\
\hline 81_09_PL & Genebank:HQ204803 & LTR 30_10 & Genebank:HQ204820 \\
\hline 82_09_PL & Genebank:HQ204804 & LTR 42_09 & Genebank:HQ204821 \\
\hline 29_07_PL & Genebank:HQ204805 & LTR 45_08 & Genebank:HQ204822 \\
\hline 31_07_PL & Genebank:HQ204806 & LTR 45_09 & Genebank:HQ204823 \\
\hline 32_07_PL & Genebank:HQ204807 & LTR 51_09 & Genebank:HQ204824 \\
\hline 2_08_PL & Genebank:HQ204808 & LTR 82_09 & Genebank:HQ204825 \\
\hline
\end{tabular}

substitutions were also detected in 12 strains without influencing the amino-acid composition of MEQ. Nucleotide alignment with $m e q$ sequences accessible in Genebank was performed. DNA sequences of meq shared $78.9 \%$ homolology of gene sites.

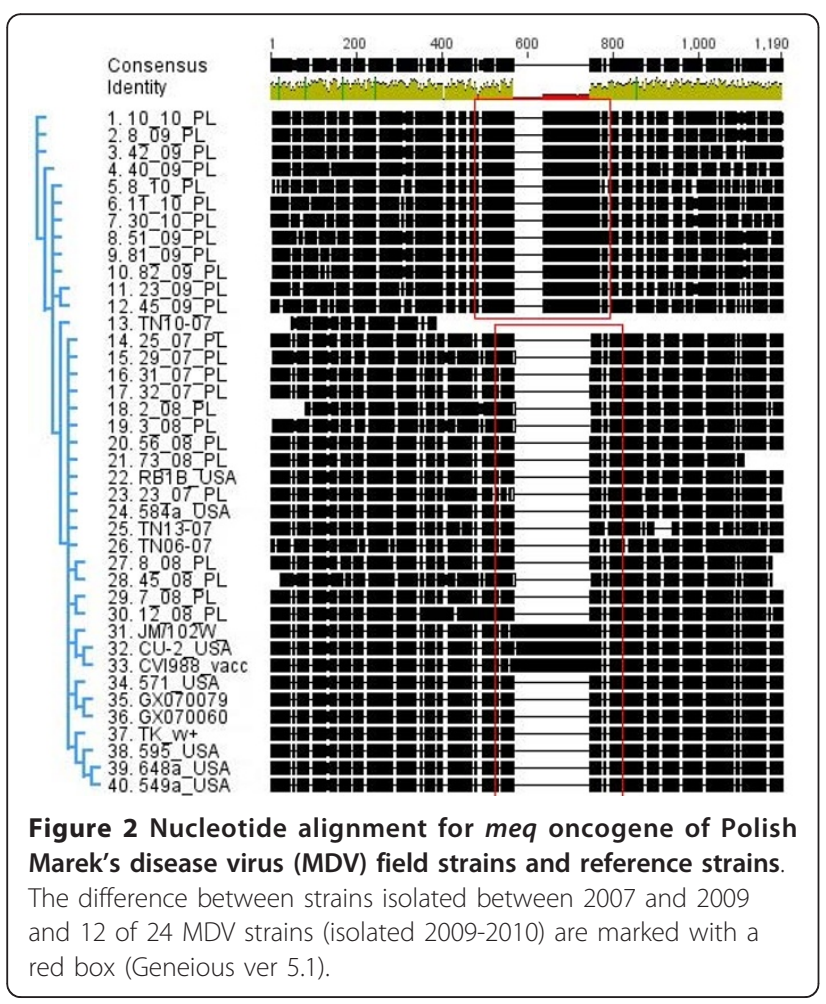

LTR analysis

PCR for LTR of REV was specific for DNA extracted from CEKs infected with the REV-T strain and showed the presence of an about $0.8 \mathrm{~kb}$ insertion in $28 \mathrm{MDV}$ strains (Figure 3). The fragment was not observed in two strains (Figure 3, lanes 14 and 30). An additional insertion of LTR-REV about $0.78 \mathrm{~kb}$ into the MDV genome was detected in two strains (Figure 3, lanes 4 and 16). Two other strains showed the presence of an additional $1.6 \mathrm{~kb}$ PCR product (Figure 3, lanes 11 and 12). Moreover in two strains (Figure 3, lanes 10 and 13) the LTR insertion was 20 bp longer than in other strains harboring the $0.8 \mathrm{bp}$ fragment. Except for the $0.8 \mathrm{~kb}$ product in DNA of the 21 strains, a fine PCR ladder of PCR products ranging from about $0.8 \mathrm{~kb}$ to $2.0 \mathrm{~kb}$ was observed in the background.

LTR fragments were sequenced and submitted to NCBI Genebank. The accession numbers are given in Table 2. The sequences were aligned with other REV sequences accessible in NCBI Genebank.

Sequencing of 10 out of 28 MDV recombinant samples confirmed the finding that MDV strains occurring in Poland have a LTR-REV insertion. These sequences shared $70.1 \%$ of LTR-REV sequence homology. An alignment of MDV-LTR fragments is shown on Figure 4. Inserts of two chosen strains were significantly longer than in case of the other 8 strains.

\section{Discussion}

Our previous study on the molecular characteristics of Polish MDV strains isolated during 2007-2009 demonstrated a close correlation between changes in nucleotide and amino-acid sequences and the strain virulence [10]. The present study expands the knowledge on the molecular characteristics of Polish MDV strains. The obtained results from sequencing of genes in 24 strains suggested a possible frame shift being detected in 12 of the recently isolated field strains. Interestingly, these strains were mainly found in chickens from the south-western parts of Poland, where cases of chicken lymphomas have been observed in the past [personal observation E SamorekSalamonowicz]. Strains not having a frame shift in their genome originated from the western regions of Poland close to the German border. The incidence of REV infections in Poland is unknown. However it is assumed, e.g. based on veterinary reports that the incidence is higher in southern Poland where poultry farming is most intensive. It is known that MDV may co-exist with other retroviruses, especially ALV and REV in chicken cells $[1,5,6]$. In vitro recombination of these viruses and MDV in chicken and duck embryo fibroblasts has been reported $[4,8]$. The 3 passages of the strains isolated from chickens with clinical MD revealed a persistent presence of LTR in the MDV genomes. A stable integration 


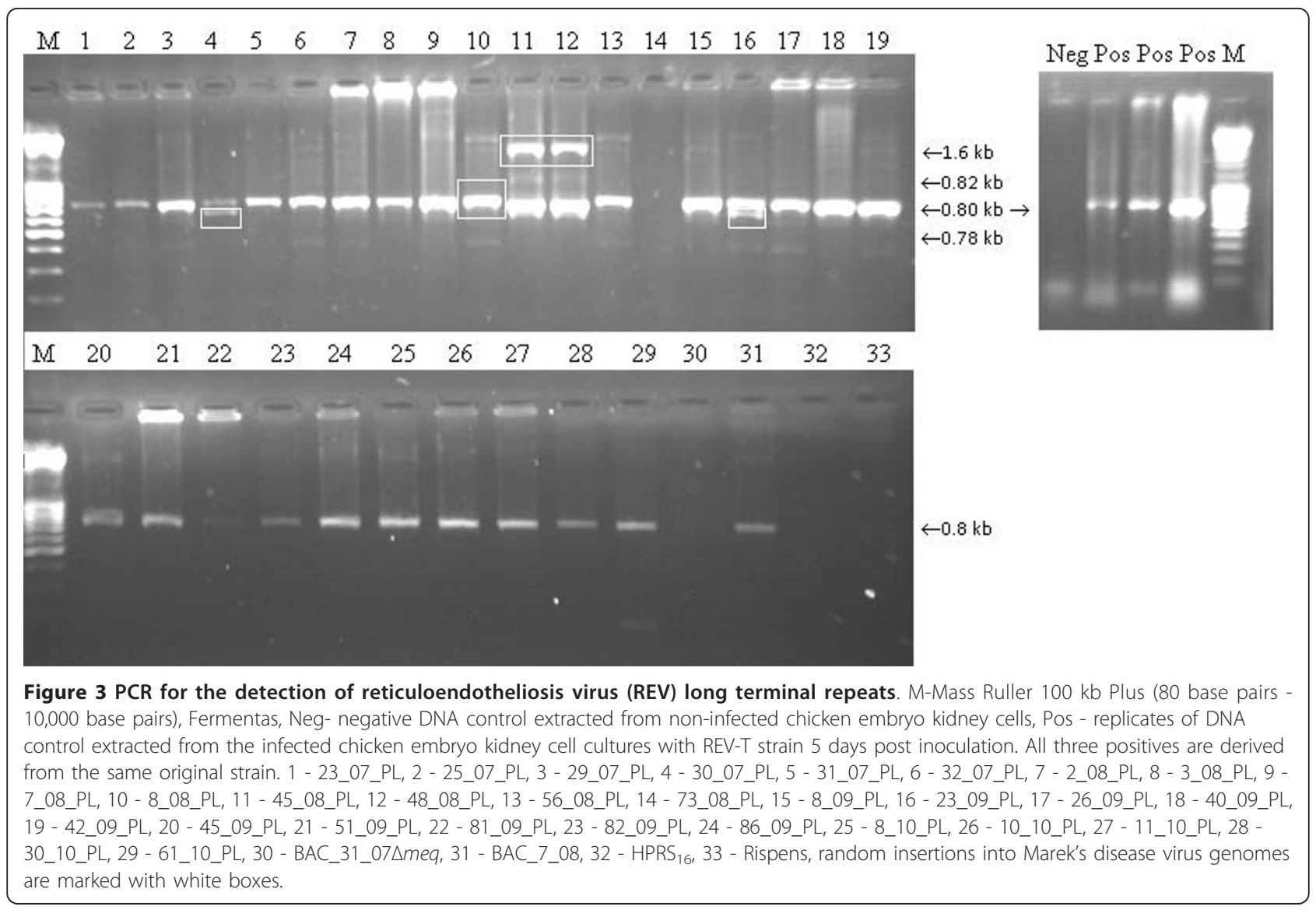

between MDV and REV is possible as previously discussed by Jones et al. [4]. In vivo studies on the molecular and biological features of MDV-REV BAC clone GX0101 and its virulence for chickens were studied by Sun et al. $[13,14]$. However the consequences of a LTR insertion into the IRs and Us junction of MDV still remains unknown. The presented study of Polish MDV strains was done using heterogeneous isolates. The obtained two BACs constructed from these strains will be a good model for further study of the effects of these insertions. In spite of detection of parallel changes in $m e q$ and LTR insertions into the MDV genome, their role remains

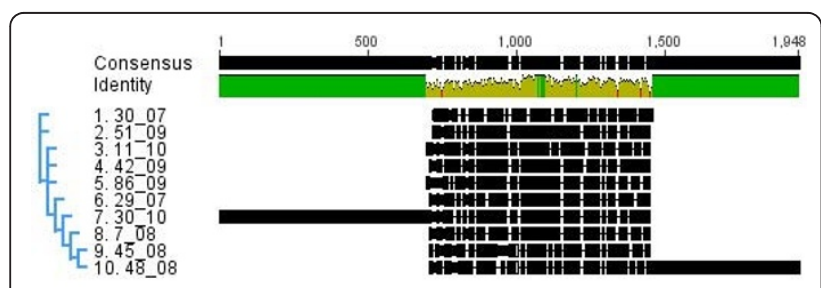

Figure 4 Nucleotide alignment of long terminal repeat inserts from 10 Polish Marek's disease virus field strains (Geneious ver 5.1). unexplained. However, LTR insertions may affect genes of importance for MDV infection mechanisms. We will investigate this in future studies using BACs. The insertions present in 12 of the 24 Polish field strains suggest that this is a common event in the high density poultry industry. In this study real-time PCR for the detection of the MDV specific amplicons like ICP4, pp38 and a part of $m e q$ revealed no influence of LTR insertion on the molecular features of these genes. However LTR insertions were also present 100-200 bp downstream from the ICP4 gene [15]. Therefore a LTR insertion may alter also basis transactivation function by ICP4. Moreover, the full sequencing of meq oncogene conducted in our study and the analysis of the assembled sequences showed a $68 \mathrm{bp}$ mutation within this gene. The insertion was located at the nucleotide 565 of meq. Similarly, in the same position a longer $180 \mathrm{bp}$ insertion was observed in attenuated strains (CU-2, JM/102W and CVI988 Rispens). Interestingly, MDV strains with the $68 \mathrm{bp}$ insertion were isolated in the south-western provinces of Poland where retroviral infections in poultry have been recognized previously. It is however possible that the phenomena occurred independently. But it might also suggest an association of the insertion with changes in virulence. The virulence of the 
isolated MDV strains were not determined in this study but will be examined in the future. However a rather high level of virulence is expected as the strains were isolated from chickens with severe clinical symptoms and MD lesions.

The $0.8 \mathrm{~kb}$ LTR insertion into the MDV genome was detected in 28 strains while 2 strains also had an insertion being visible as a $1.6 \mathrm{~kb}$ band. This insertion could be assigned as a most efficiently transcribed LTR region within SORF2 region of MDV [4]. SORF2 is a homologue of US22 gene of human cytomegalovirus and human herpesvirus 6 EPLF3 $[4,16]$. This insertion may alter transactivation of MDV genes as suggested in previous studies $[8,9]$. The multiple bands observed in a few Polish field strains suggest different sites of LTR integration in the $\mathrm{U}_{\mathrm{S}} \mathrm{MDV}$ region. Jones et al. [8] described smeared bands as randomly integrated REV proviruses. This may influence the sequence and structure of different MDV genes including meq. However the smears in the background of the deletive BAC_31_07 $\Delta m e q$ suggest that the insertions are not necessarily associated only with meq (Figure 1 ). The sequenced LTR fragments of 10 strains were new in NCBI Genebank. The changes detected within meq are confirmed by the finding that LTR insertions are often located near to proto-oncogenes thus being able to activate tumoral processes [3]. An other scenario is that $I_{S}$ and $U_{s}$ insertions may alter the recombination of chimeric viruses in vitro and in vivo since the LTR-REV regions are known as strong enhancers of promoters in chicken embryo fibroblasts [17]. However confirmation of these hypotheses needs further studies. Previous reports on the effects of a LTR-REV insertion into RM1 [1] or GX0101 [13,14] strains of MDV suggest attenuation and reduction of their oncogenicity with intact replication abilities. However insertions in Polish MDV strains seemed not to influence their oncogenicity since they caused outbreaks of MD in vaccinated chickens. The exact effects of these insertions will be studied experimentally after establishing BAC clones lacking the LTR fragment. The LTR insertions in MDV could make the epidemiology of Marek's disease more complex. These aspects will be investigated in future studies.

\section{Conclusions}

Significant insertions within meq oncogene sequence were detected among recently isolated virulent MDV strains from Poland. The detected insertional mutation may lead to structural and functional changes of multiple proteins and virus features.

\section{List of abbreviations}

ALV: Avian leukosis virus; BAC: bacterial artificial chromosome including MDV genome; bp: base pair; CEK: chicken embryo kidney cells; DMSO: dimethyl sulfoxide; EPLF3: gene of human herpesvirus 6 ; $I R_{L}$ : internal repeated long sequence of $M D V ; \quad I R_{s}$ : internal repeated short sequence of $M D V ; k b p$ : thousand of base pairs; LTR: long terminal repeats of REV genome; LTZ: Lohmann Tierzucht Company, Germany; MEM: minimal essential medium; MEQ: main oncoprotein of MDV crucial in tumorigenesis; MDV: Marek's disease virus; REV: reticuloendotheliosis virus; SPF: specific pathogen free chickens. The following pathogens are certified to be absent: avian adenoviruses, infectious bronchitis virus, infectious laryngotracheitis virus, avianleukosis, avian reticuloendotheliosis and Marek's disease virus; SORF2: short opened reading frame 2 of MDV; TRs: terminal repeated short sequence of MDV; TR: terminal repeated long sequence of MDV; $U_{s}$ : unique short region of $M D V ; U_{L}$ : unique long region of MDV; US22: gene located within unique short region of MDV; ver. -version.

\section{Acknowledgements}

Dr Peter Biggs, Houghton Poultry Research Station, Huntingdon, UK is acknowledged for providing reference strain $\mathrm{HPRS}_{16}$.

\section{Authors' contributions}

GW carried out isolation of the used strains, DNA extraction, PCRs, sequencing of mea and LTR genes and nucleotide alignments. ESS and WK participated in DNA extraction, real-time PCRs, PCRs and assesment of the final results. ESS provided personal observations used in the present study. WK provided detailed information regarding origin of used MDV field strains. ESS and WK participated in coordination of the study. The final manuscript was read and approved by all authors.

\section{Competing interests}

The authors declare that they have no competing interests.

Received: 24 November 2010 Accepted: 14 February 2011 Published: 14 February 2011

\section{References}

1. Witter RL, Li D, Jones D, Lee LF, Kung HJ: Retroviral insertional mutagenesis of a herpesvirus: a Marek's disease virus mutant attenuated for oncogenicity but not for immunosuppression or in vivo replication. Avian Dis 1997, 41:407-421.

2. Woźniakowski G, Samorek-Salamonowicz E, Kozdruń W: Occurence of main Marek's disease genes during infection of chickens. Bull Vet Inst Pulawy 2010, 54:123-127.

3. Isfort R, Jones D, Kost R, Witter R, Kung HJ: Retrovirus insertion into herpesvirus in vitro and in vivo. Proc Natl Acad Sci USA 1992, 89:991-995.

4. Jones D, Brunovskis $P$, Witter R, Kung HJ: Retroviral insertional activation in a herpesvirus: transcriptional activation of US genes by an integrated long terminal repeat in a Marek's disease virus clone. J Virol 1996, 70:2460-2467.

5. Zhang Z, Cui Z: Isolation of recombinant field strains of Marek's disease virus integration with reticuloendotheliosis virus genome fragments. Sci China C Life Sci 2005, 48:81-88.

6. Davidson I, Borenshtain R, Kung HJ, Witter RL: Molecular indications for in vivo integration of the avian leukosis virus, subgroup J-long terminal repeat into the Marek's disease virus in experimentally dually-infected chickens. Virus Genes 2002, 24:173-180.

7. Witter RL, Fadly AM: Neoplastic diseases: Reticuloendotheliosis. In Diseases of poultry. 12 edition. Edited by: Saif YM, Fadly AM, Glisson JR, McDougald LR, Nolan LK, Swayne DE. Ames, USA; 2008:515-529.

8. Jones $D$, Isfort $R$, Witter $R$, Kost R, Kung HJ: Retroviral insertions into a herpesvirus are clustered at the junctions of the short repeat and short unique sequences. Proc Natl Acad Sci USA 1993, 90:3855-3859.

9. Davidson I, Borenshtain $\mathrm{H}$ : In vivo events of retroviral long terminal repeat integration into Marek's disease virus in commercial poultry: detection of chimeric molecules as a marker. Avian Dis 2001, 45:102-121.

10. Woźniakowski G, Samorek-Salamonowicz E, Kozdruń W: Sequence analysis of meq oncogene among Polish strains of Marek's disease. Pol J Vet Sci 2010, 13:263-267.

11. Hauck R, Prusas C, Hafez HM, Lüschow D: Quantitative PCR as a tool to determine the reticuloendotheliosis virus-proviral load of fowl poxvirus. Avian Dis 2009, 53:211-215.

12. Drummond AJ, Ashton B, Buxton S, Cheung M, Cooper A, Heled J, Kearse M, Moir R, Stones-Havas S, Sturrock S, Thierer T, Wilson A: Geneious ver. 5.1. [http://www.geneious.com]. 
13. Sun A, Petherbridge L, Zhao Y, Li Y, Nair VK, Cui Z: A BAC clone of MDV strain GX0101 with REV-LTR integration retained its pathogenicity. Chin Sci Bull 2010, 54:2641-2647.

14. Sun $A$, Xu X, Petherbridge L, Zhao Y, Nair V, Cui Z: Functional evaluation of the role of reticuloendotheliosis virus long terminal repeat (LTR) integrated into the genome of a field strain of Marek's disease virus. Virology 2010, 397:270-276

15. Sakaguchi M, Urakawa T, Hirayama Y, Miki N, Yamamoto M, Hirai K: Sequence determination and genetic content of an 8.9-kb restriction fragment in the short unique region and the internal inverted repeat of Marek's disease virus type 1 DNA. Virus Genes 1992, 6:365-378.

16. Stasiak PC, Mocarski EC: Transactivation of the cytomegalovirus ICP36 gene promoter requires the alpha gene product TRS1 in addition to IE1 and IE2. J Virol 1992, 66:1050-1058

17. Hirano A, Wong T: Functional interaction between transcriptional elements in the long terminal repeat of reticuloendotheliosis virus: cooperative DNA binding of promoter- and enhancer-specific factors. Mol Cell Biol 1999, 8:5232-5244

doi:10.1186/1751-0147-53-10

Cite this article as: Woźniakowski et al:: Molecular characteristics of Polish field strains of Marek's disease herpesvirus isolated from vaccinated chickens. Acta Veterinaria Scandinavica 2011 53:10.

\section{Submit your next manuscript to BioMed Central} and take full advantage of:

- Convenient online submission

- Thorough peer review

- No space constraints or color figure charges

- Immediate publication on acceptance

- Inclusion in PubMed, CAS, Scopus and Google Scholar

- Research which is freely available for redistribution

Submit your manuscript at www.biomedcentral.com/submit 